(C) IEEE

I. Iriarte, E. Otaola, D. Culla, I. Iglesias, J. Lasa and B. Sierra, "Modeling and control of an overactuated aerial vehicle with four tiltable quadrotors attached by means of passive universal joints," 2020 International Conference on Unmanned Aircraft Systems (ICUAS), Athens, Greece, 2020, pp. 1748-1756, doi:

10.1109/ICUAS48674.2020.9213848.

https://doi.org/10.1109/icuas48674.2020.9213848 


\title{
Modeling and control of an overactuated aerial vehicle with four tiltable quadrotors attached by means of passive universal joints
}

\author{
Imanol Iriarte*, Erlantz Otaola*, David Culla*, Iñaki Iglesias*, \\ Joseba Lasa* and Basilio Sierra**
}

\begin{abstract}
We present a novel overactuated aerial vehicle based on four quadrotors connected to an airframe by means of passive universal joints. The proposed architecture allows to independently control the six degrees of freedom of the airframe without having fixed propellers at inefficient configurations or making use of dedicated rotor tilting actuators. After deriving the dynamic equations that describe its motion, we propose a linear control strategy that is able to successfully decouple rotation and translation, relying exclusively on on-board sensors. A prototype is built and preliminary experimental results demonstrate that the concept is feasible.

Video: https://youtu.be/9ASP3FyhCJw
\end{abstract}

\section{INTRODUCTION}

Multirotor aerial vehicles are receiving increasing attention, not only from the research community, but also from the industry and the wide public. Their ability to reach the skies with no need of infrastructure, high maneuverability and lower cost, noise, emissions and complexity than conventional helicopters has made them useful in applications such as search and rescue operations, infrastructure inspection, traffic monitoring, agriculture or filming. While most of these tasks exclusively make use of the sensing capabilities of the vehicles, physical interaction of aerial vehicles with their environment and with humans is currently being investigated in fields like aerial manipulation (see [1] and [2]) and it could broaden the scope of this technology to activities such as work in hazardous conditions, natural disaster cleanup, remote infrastructure maintenance, pick and place operations or even last-mile logistics and human transportation.

Parallel axis multirotors, where all the rotors are aligned in the same direction, have been useful in many applications because they are mechanically simple and maximize the amount of thrust generated along a single direction, increasing payload and flight time. However, parallel axis multirotors are underactuated vehicles: they can not simultaneously generate forces and torques in the three spatial dimensions, which makes it impossible to independently control their six degrees of freedom. This lack of maneuverability becomes a strong handicap when complex interaction with the environment is required.

*The authors are with the Tecnalia Electric Aircraft Lab, Tecnalia Research and Innovation, Paseo Mikeletegi 7, 20009 Donostia-San Sebastián, Spain. \{imanol.iriarte, erlantz.otaola, david.culla, inaki.iglesias, joseba.lasa\}@tecnalia.com

**The author is with the Robotics and Autonomous Systems Group (RSAIT), University of the Basque Country (UPV-EHU), Manuel Lardizabal 1, 20018 Donostia-San Sebastián, Spain. b. sierra@ehu.eus

This research was supported by the ELKARTEK 2018 program of the Basque Government, grant agreement No. KK-2018/00082.

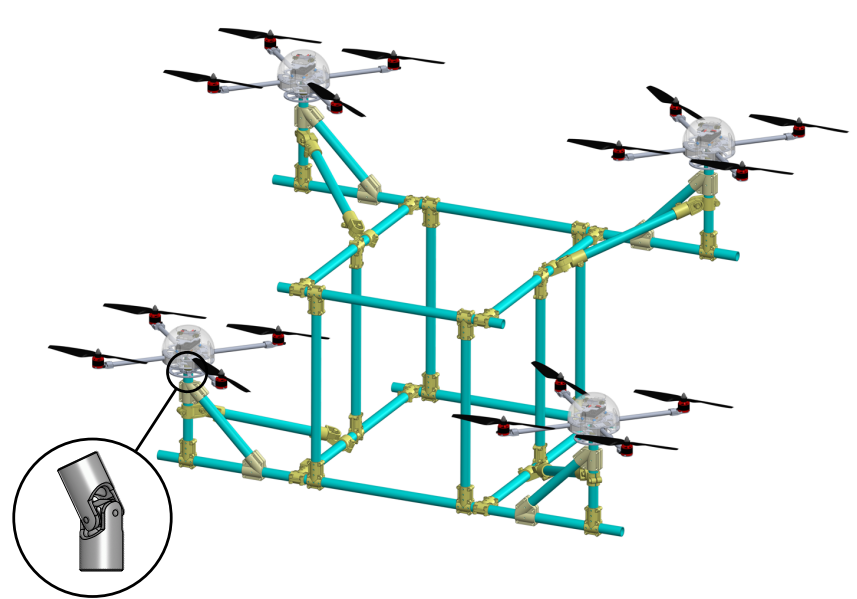

Fig. 1. Render of the proposed architecture: four quadrotors connected to an airframe by means of passive universal joints.

\section{A. Related work}

Underactuation has frequently been tackled by attaching complex mechanisms such as gimbals, robotic arms or parallel manipulation systems to parallel axis multirotors [3]-[7]. Such a strategy increases the weight and generates a complex control problem, because the dynamics of the vehicle are coupled with those of the mechanism. Another way of overcoming underactuation involves designing new multirotor architectures, which can be classified according to their propeller tilting mechanism in the following three groups: fixed tilting, active tilting and passive tilting.

In the first group, overactuation is achieved by attaching rotors to an airframe with a fixed tilting angle. For instance, in [8]-[12], some hexarotors are presented and in [13] one with variable pitch propellers is proposed. In [14], a passive robotic arm is attached to a fixed tilting hexarotor, enabling manipulation with reduced system complexity and weight. The octorotors designed in [15] and [16] have half of the propellers arranged in the horizontal plane and another set of four smaller propellers located perpendicularly to that plane, to exert forces in the two remaining directions. The authors of [17] design a novel multirotor architecture by solving a technical optimization problem. Similarly, in [18] and [19], vehicle topology is optimized to achieve omnidirectional force and torque exertion by means of bidirectional propellers and [20] studies the theoretical conditions to develop omnidirectional vehicles based on unidirectional propellers with fixed tilting. The same approach can be applied to the collaborative transportation of objects: while attaching 
several quadrotors to a given payload in the same plane leads to underactuated dynamics [21], the introduction of fixed tilting angles makes the system overactuated [22]. Fixed tilting angle multirotors are mechanically simple but tend to be energetically inefficient because part of the thrust produced by propellers is lost generating internal forces.

The second group includes vehicles in which dedicated actuators are used to control the tilting of the rotors. In [23], a trirotor with active tilting of two of its rotors is presented: even if still underactuated, it can control one degree of freedom more than parallel axis trirotors. An aerial vehicle based on two coaxial counter-rotating propellers and a set of three ducted fans whose tilting angle is actively controlled is described in [24]. The hexarotor presented in [25] uses a single actuator to simultaneously modify the tilting angle of all propellers, varying from an underactuated configuration to a maximally actuated one. A more complex setup, where the tilting of each propeller is independently controlled, is applied to a quadrotor in [26] and [27] and to an hexarotor in [28]. The authors of [29] propose a vehicle in which two tilting angles are independently controlled for each propeller. Similarly, in [30] and [31] only two actuators are used to simultaneously control the two tilting angles of all propellers. Active tilting multirotors can avoid some of the inefficiencies of fixed tilting angle multirotors by better aligning thrust, but at the cost of increased system complexity and weight, due to the addition of extra actuators.

Eventually, passive tiliting multirotors are vehicles with the ability to orientate their rotors without making use of dedicated tilting actuators. This can be achieved through collaborative setups, where multiple underactuated vehicles are attached to a single body via passive mechanisms like cables [32]-[36], rigid rods [37], [38] or spherical joints [39], [40]. These modular architectures can control propeller orientation to align all the thrust along the desired direction and avoid inefficient internal forces. Besides, the absence of dedicated rotor tilting actuators reduces vehicle weight, complexity, energy consumption and probability of failure.

Spherical joints allow three rotational degrees of freedom between the bodies they connect and, ideally, they exclusively transmit forces, thus, spherically joined quadrotors act as orientable thrust generators. However, in order to orientate a vector in space, two rotational degrees of freedom are enough. Hence, we propose an architecture based on universal joints (see Figure 1). Universal joints only allow two rotational degrees of freedom and they transmit force and torque, turning the quadrotors into orientable thrust and torque generators. Each universal joint has one degree of freedom less to control and it provides one control variable more than a spherical joint, reducing the complexity of the control problem.

\section{B. Contributions}

This paper makes the following contributions:

- Introduction of what, to our knowledge, is the first overactuated aerial vehicle based on multiple quadrotors connected to an airframe by means of universal joints.

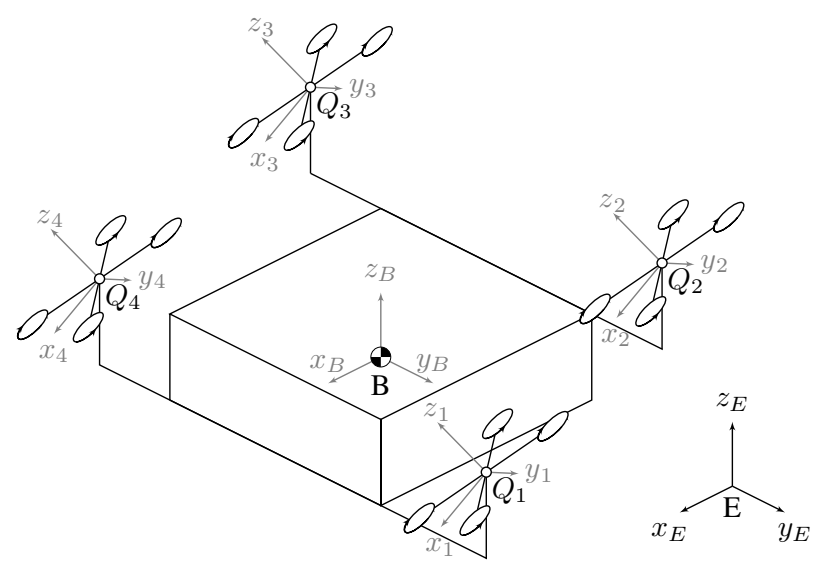

Fig. 2. System architecture and frames of reference.

- Derivation with the Newton-Euler method of the dynamic equations that describe the vehicle's motion.

- Description of a linear control strategy that is able to successfully decouple rotation and translation, relying exclusively on on-board sensors.

- Presentation of preliminary experimental results that show the feasibility of the concept.

\section{Structure}

This document is structured as follows: section II derives the equations of motion of the system, section III describes the adopted control strategy, section IV presents some experimental results and section $\mathrm{V}$ discusses the conclusions of this paper and future research topics.

\section{System Modeling}

\section{A. Notation}

All along this paper vectors will be denoted by lowercase bold letters (e.g. $\boldsymbol{a}$ ) and matrices by uppercase bold letters (e.g. A). Vector $\boldsymbol{a}$ expressed in a frame of reference $\mathcal{F}_{X}$ is denoted as $\{\boldsymbol{a}\}_{X}$, matrix $\mathbf{A}$ expressed in frame $\mathcal{F}_{X}$ is denoted as $[\mathbf{A}]_{X} .\{\boldsymbol{a}\}_{X}$ can be transformed into frame of reference $\mathcal{F}_{Y}$ left multiplying it with rotation matrix ${ }^{Y} \mathbf{R}_{X}$

$$
\{\boldsymbol{a}\}_{Y}={ }^{Y} \mathbf{R}_{X}\{\boldsymbol{a}\}_{X} .
$$

The whole system can be mathematically represented as a rigid solid linked by means of universal joints to four quadrotors. In order to describe its motion, six frames of reference will be used: Earth $\mathcal{F}_{E}\left(E, x_{E}, y_{E}, z_{E}\right)$, inertial; Body $\mathcal{F}_{B}\left(B, x_{B}, y_{B}, z_{B}\right)$, attached to the center of mass of the platform; and Quadrotor $\mathcal{F}_{Q_{i}}\left(Q_{i}, x_{i}, y_{i}, z_{i}\right), i=1 \ldots 4$, attached to the center of mass of each quadrotor, which is assumed to exactly coincide with the center of rotation of the universal joint (see Figure 2).

Let the translational position of the center of mass expressed in $\mathcal{F}_{E}$ be $\{\boldsymbol{r}\}_{E}=\left\{r_{\mathrm{X}}, r_{\mathrm{Y}}, r_{\mathrm{Z}}\right\}_{E}^{\mathrm{T}}$ and the rotational speed expressed in $\mathcal{F}_{B}\{\boldsymbol{\omega}\}_{B}=\left\{\omega_{\mathrm{x}}, \omega_{\mathrm{y}}, \omega_{\mathrm{z}}\right\}_{B}^{\mathrm{T}}$. The airframe attitude with respect to Earth is defined by the ZYX TaitBryan angles: yaw $(\psi)$, pitch $(\theta)$ and roll $(\phi), \boldsymbol{\eta}=\{\phi, \theta, \psi\}^{\mathrm{T}}$. This orientation can be represented as a rotation matrix, 


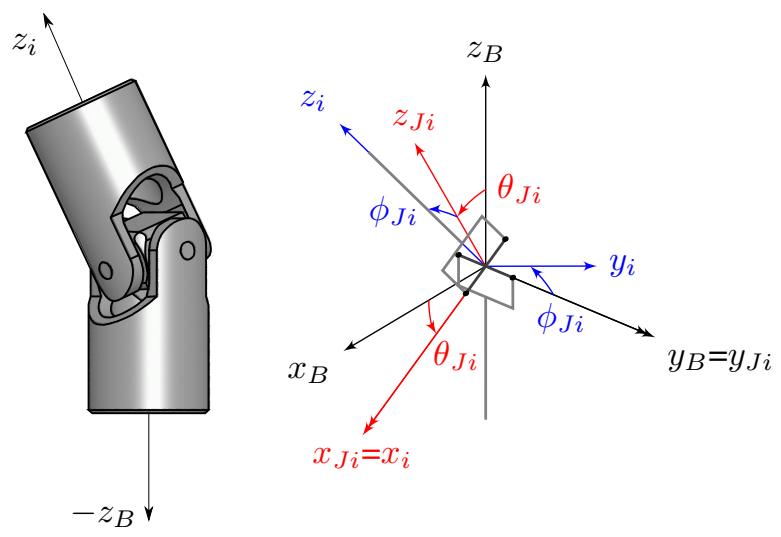

Body $\underset{y_{B}=y_{J i}}{\stackrel{\theta_{J i}}{\longrightarrow}}$ Joint $\underset{x_{J i}=x_{i}}{\stackrel{\phi_{J i}}{\longrightarrow}}$ Quadcopter $i$

Fig. 3. Mathematical representation of the universal joint: only two degrees of freedom between the connected bodies are allowed: rotations $\phi_{J i}$ and $\theta_{J i}$.

obtained from the multiplication of three basic rotation matrices [41]

$$
{ }^{E} \mathbf{R}_{B}=\left[\begin{array}{ccc}
\mathrm{c}_{\theta} \mathrm{c}_{\psi} & \mathbf{s}_{\phi} \mathbf{s}_{\theta} \mathrm{c}_{\psi}-\mathrm{c}_{\phi} \mathbf{s}_{\psi} & \mathbf{c}_{\phi} \mathbf{s}_{\theta} \mathrm{c}_{\psi}+\mathbf{s}_{\phi} \mathbf{s}_{\psi} \\
\mathrm{c}_{\theta} \mathbf{s}_{\psi} & \mathbf{s}_{\phi} \mathbf{s}_{\theta} \mathbf{s}_{\psi}+\mathbf{c}_{\phi} \mathbf{c}_{\psi} & \mathbf{c}_{\phi} \mathbf{s}_{\theta} \mathrm{s}_{\psi}-\mathbf{s}_{\phi} \mathbf{c}_{\psi} \\
-\mathbf{s}_{\theta} & \mathbf{s}_{\phi} \mathbf{c}_{\theta} & \mathbf{c}_{\phi} \mathbf{c}_{\theta}
\end{array}\right],
$$

where $\mathrm{s}_{\theta}$ and $\mathrm{c}_{\theta}$ denote $\sin \theta$ and $\cos \theta$ respectively and similarly for $\phi$ and $\psi$. The rotational speed expressed in $\mathcal{F}_{B}$ is related to the time derivative of the Tait-Bryan angles $\dot{\boldsymbol{\eta}}=\{\dot{\phi}, \dot{\theta}, \dot{\psi}\}^{\mathrm{T}}$ by [42]:

$$
\{\boldsymbol{\omega}\}_{B}=\left[\begin{array}{ccc}
1 & 0 & -\mathrm{s}_{\theta} \\
0 & \mathrm{c}_{\phi} & \mathrm{s}_{\phi} \mathrm{c}_{\theta} \\
0 & -\mathrm{s}_{\phi} & \mathrm{c}_{\phi} \mathrm{c}_{\theta}
\end{array}\right] \dot{\boldsymbol{\eta}}
$$

Each quadrotor's position with respect to the Body is defined by vector $\left\{\boldsymbol{l}_{i}\right\}_{B}=\left\{l_{x i}, l_{y i}, l_{z i}\right\}_{B}^{\mathrm{T}}$ and its angular velocity by $\left\{\boldsymbol{\omega}_{i}\right\}_{Q i}=\left\{\omega_{\mathrm{x} i}, \omega_{\mathrm{y} i}, \omega_{\mathrm{zi}}\right\}_{Q i}^{\mathrm{T}}$. Universal joints only allow two rotational degrees of freedom $\left(\theta_{J i}\right.$ and $\left.\phi_{J i}\right)$ between the bodies they connect (see Figure 3).

Hence, the orientation of each quadrotor with respect to Body can be expressed by means of rotation matrix ${ }^{B} \mathbf{R}_{Q_{i}}\left(\boldsymbol{\eta}_{J i}\right)$, being $\boldsymbol{\eta}_{J i}=\left\{\phi_{J i}, \theta_{J i}, 0\right\}^{\mathrm{T}}$ the relative TaitBryan angles or, equivalently, with Earth referenced angles $\boldsymbol{\eta}_{i}=\left\{\phi_{i}, \theta_{i}, \psi_{i}\right\}^{\mathrm{T}}$, satisfying that

$$
{ }^{E} \mathbf{R}_{Q_{i}}\left(\boldsymbol{\eta}_{i}\right)={ }^{E} \mathbf{R}_{B}(\boldsymbol{\eta})^{B} \mathbf{R}_{Q_{i}}\left(\boldsymbol{\eta}_{J i}\right) .
$$

The vehicle has total mass $\mathrm{m}$. The airframe's inertia is $\mathbf{I}^{B} \in \mathbb{R}^{3 \times 3}$, the quadrotors' inertia (quadrotor frame and propellers) is denoted by $\mathbf{I}^{Q} \in \mathbb{R}^{3 \times 3}$ and the propellers' inertia by $\mathbf{I}^{P} \in \mathbb{R}^{3 \times 3}$.

Rotational speed of propeller $j=1 \ldots 4$ from quadrotor $i=1 \ldots 4$ is denoted as $n_{i j}$. Each propeller's position with respect to $Q_{i}$ is determined by vector $\mathbf{d}_{i j} \in \mathbb{R}^{3 \times 1}$ of modulus $\mathrm{d}$ and it produces a force $\boldsymbol{f}_{i j} \in \mathbb{R}^{3 \times 1}$ and a torque $\boldsymbol{\tau}_{i j} \in \mathbb{R}^{3 \times 1}$ along the $z_{i}$ direction.

It is convenient to make a variable transformation between the forces and torques produced by propellers and variables $\boldsymbol{u}_{h i}, h=1, \ldots, 4$, named control actions (see Figure 4,
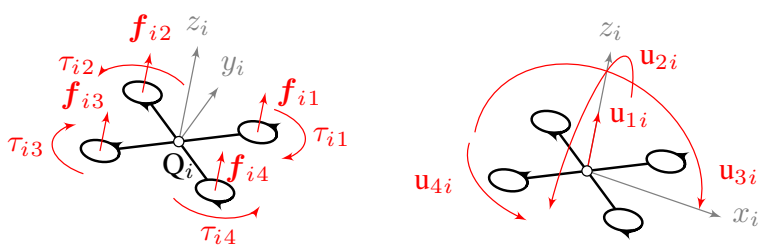

Fig. 4. Variable transformation: the forces and torques produced by each propeller are replaced by quadrotor thrust and roll, pitch and yaw torques.

where $\boldsymbol{u}_{1 i}$ represents the total thrust of the $i$-th quadrotor, $\boldsymbol{u}_{2 i}$ the roll torque, $\boldsymbol{u}_{3 i}$ the pitch torque and $\boldsymbol{u}_{4 i}$ the yaw torque.

$$
\begin{aligned}
& u_{1 i}=\left(f_{i 1}+f_{i 2}+f_{i 3}+f_{i 4}\right) \\
& u_{2 i}=\left(f_{i 1}+f_{i 2}-f_{i 3}-f_{i 4}\right) \frac{\sqrt{2}}{2} \mathrm{~d} \\
& u_{3 i}=\left(f_{i 2}+f_{i 3}-f_{i 1}-f_{i 4}\right) \frac{\sqrt{2}}{2} \mathrm{~d} \\
& u_{4 i}=-\tau_{i 1}+\tau_{i 2}-\tau_{i 3}+\tau_{i 4}
\end{aligned}
$$

\section{B. Simplifying assumptions}

In order to keep the dynamic model of the system tractable, some simplifications will be considered:

- Each quadrotor's center of mass coincides with the rotational center of the universal joint.

- All quadrotors are assumed to have identical physical parameters.

- All the bodies are considered rigid.

- It is assumed that $\phi_{J i}$ and $\theta_{J i}$ are small and never reach saturation of the universal joint.

- The system is symmetric and propellers are represented as flat, uniform discs. Thus, inertia tensors of airframe, quadrotor and propeller are assumed diagonal when referenced to their center of mass and expressed in their fixed frame of reference.

- The thrust force and the reaction torque of a propeller are proportional to its angular velocity squared and transitory dynamics are neglected

$$
\begin{aligned}
& f_{i j}=k_{f} n_{i j}^{2} \\
& \tau_{i j}=k_{\tau} n_{i j}^{2} .
\end{aligned}
$$

Hence, the control actions can be expressed as a function of the propeller angular speeds

$$
\left\{\begin{array}{l}
u_{1 i} \\
u_{2 i} \\
u_{3 i} \\
u_{4 i}
\end{array}\right\}=\left[\begin{array}{cccc}
k_{f} & k_{f} & k_{f} & k_{f} \\
\frac{\sqrt{2}}{2} \mathrm{~d} k_{f} & \frac{\sqrt{2}}{2} \mathrm{~d} k_{f} & -\frac{\sqrt{2}}{2} \mathrm{~d} k_{f} & -\frac{\sqrt{2}}{2} \mathrm{~d} k_{f} \\
\frac{\sqrt{2}}{2} \mathrm{~d} k_{f} & \frac{\sqrt{2}}{2} \mathrm{~d} k_{f} & -\frac{\sqrt{2}}{2} \mathrm{~d} k_{f} & -\frac{\sqrt{2}}{2} \mathrm{~d} k_{f} \\
-k_{\tau} & k_{\tau} & -k_{\tau} & k_{\tau}
\end{array}\right]\left\{\begin{array}{l}
n_{i 1}^{2} \\
n_{i 2}^{2} \\
n_{i 3}^{2} \\
n_{i 4}^{2}
\end{array}\right\}
$$

- Aerodynamic drag forces and torques are neglected.

- Interfering air flows are not considered.

\section{Equations of motion}

The proposed architecture has fourteen degrees of freedom and its motion can be fully described by means of three translational coordinates $\left(x_{E}, y_{E}, z_{E}\right)$, three airframe rotational coordinates $(\phi, \theta, \psi)$ and eight quadrotor angular coordinates $\left(\phi_{i}, \theta_{i}\right), i=1, \ldots, 4$.

The Newton-Euler procedure is followed to model the dynamics of the system. Unlike spherical joints, universal 
joints block relative yaw $\left(\psi_{J i}=0\right)$ by means of constraint torque $\tau_{J i}$ along the $z_{J i}$ axis (see Figure 3)

$$
\begin{aligned}
\left\{\boldsymbol{\tau}_{J_{i}}\right\}_{B} & =\left\{\tau_{J_{i}} \mathrm{~s}_{\theta_{J i}}, 0, \tau_{J i} \mathrm{c}_{\theta_{J i}}\right\}_{B}^{\mathrm{T}} \\
\left\{-\boldsymbol{\tau}_{J_{i}}\right\}_{Q i} & =\left\{0,-\tau_{J i} \mathrm{~s}_{\phi_{J i}},-\tau_{J_{i}} \mathrm{c}_{\phi_{J i}}\right\}_{Q i}^{\mathrm{T}} .
\end{aligned}
$$

1) Translational dynamics: Neglecting aerodynamic drag, the forces acting on the system are $\boldsymbol{u}_{1 i}$ and gravity $(\mathrm{mg})$. Applying Newton's second law leads to

$$
\mathrm{m} \ddot{\boldsymbol{r}}=\sum_{i=1}^{4} \boldsymbol{u}_{1 i}+\mathrm{mg}
$$

which can be expressed in $\mathcal{F}_{E}$ as

$$
\mathrm{m}\{\ddot{\boldsymbol{r}}\}_{E}=\sum_{i=1}^{4}{ }^{E} \mathbf{R}_{Q_{i}}\left\{\boldsymbol{u}_{1 i}\right\}_{Q i}+\mathrm{m}\{\boldsymbol{g}\}_{E}
$$

and can be expanded to

$$
\begin{aligned}
& \mathrm{m} \ddot{r}_{\mathrm{X}}=\sum_{i=1}^{4}\left(\mathrm{c}_{\phi_{i}} \mathrm{~s}_{\theta_{i}} \mathrm{c}_{\psi_{i}}+\mathrm{s}_{\phi_{i}} \mathrm{~s}_{\psi_{i}}\right) u_{1 i} \\
& \mathrm{~m} \ddot{r}_{\mathrm{Y}}=\sum_{i=1}^{4}\left(\mathrm{c}_{\phi_{i}} \mathrm{~s}_{\theta_{i}} \mathrm{~s}_{\psi_{i}}-\mathrm{s}_{\phi_{i}} \mathrm{c}_{\psi_{i}}\right) u_{1 i} \\
& \mathrm{~m} \ddot{r}_{\mathrm{Z}}=\sum_{i=1}^{4}\left(\mathrm{c}_{\phi_{i}} \mathrm{c}_{\theta_{i}}\right) u_{1 i}-\mathrm{mg}
\end{aligned}
$$

2) Airframe angular dynamics: Being the airframe a rigid body, Euler's second law of motion leads to the following set of equations

$$
\mathbf{I}^{B} \dot{\boldsymbol{\omega}}+\boldsymbol{\omega} \times \mathbf{I}^{B} \boldsymbol{\omega}=\sum_{i=1}^{4}\left(\boldsymbol{\tau}_{\boldsymbol{J}}+\boldsymbol{l}_{i} \times \boldsymbol{u}_{1 i}\right)
$$

which can be expressed in $\mathcal{F}_{B}$ as

$$
\begin{aligned}
& {\left[\mathbf{I}^{B}\right]_{B}\{\dot{\boldsymbol{\omega}}\}_{B}+\{\boldsymbol{\omega}\}_{B} \times\left[\mathbf{I}^{B}\right]_{B}\{\boldsymbol{\omega}\}_{B}} \\
& =\sum_{i=1}^{4}\left(\left\{\boldsymbol{\tau}_{\boldsymbol{J}_{i}}\right\}_{B}+\left\{\boldsymbol{l}_{i}\right\}_{B} \times{ }^{B} \mathbf{R}_{Q_{i}}\left\{\boldsymbol{u}_{1 i}\right\}_{Q i}\right)
\end{aligned}
$$

and ${ }^{B} \mathbf{R}_{Q_{i}}$ can be determined with Equation 4 Let the following scalars be defined as

$$
\begin{aligned}
& \alpha_{x i}=-\mathbf{s}_{\theta-\theta_{i}} \mathbf{c}_{\phi_{i}} \\
& \alpha_{y i}=\mathbf{s}_{\phi} \mathbf{c}_{\theta} \mathrm{c}_{\phi_{i}} \mathrm{c}_{\theta_{i}}-\mathrm{c}_{\phi} \mathbf{s}_{\phi_{i}}+\mathbf{s}_{\phi} \mathbf{s}_{\theta} \mathrm{c}_{\phi_{i}} \mathbf{s}_{\theta_{i}} \\
& \alpha_{z i}=\mathrm{c}_{\phi} \mathrm{c}_{\theta} \mathrm{c}_{\phi_{i}} \mathrm{c}_{\theta_{i}}+\mathrm{s}_{\phi} \mathrm{s}_{\phi_{i}}+\mathrm{c}_{\phi} \mathrm{s}_{\theta} \mathrm{c}_{\phi_{i}} \mathrm{~s}_{\theta_{i}}
\end{aligned}
$$

such that $\alpha_{x i} u_{1 i}$ is the projection of $\boldsymbol{u}_{1 i}$ in $x_{B}$ and similarly for the rest of axis. Then, the expansion of Equation 15 is

$$
\begin{aligned}
\mathrm{I}_{\mathrm{xx}}^{B} \dot{\omega}_{\mathrm{x}}= & \left(\mathrm{I}_{\mathrm{yy}}^{B}-\mathrm{I}_{\mathrm{zz}}^{B}\right) \omega_{\mathrm{y}} \omega_{\mathrm{z}} \\
& +\sum_{i=1}^{4}\left(\tau_{J i} \mathrm{~s}_{\theta_{J i}}+\left(l_{y i} \alpha_{z i}-l_{z i} \alpha_{y i}\right) u_{1 i}\right) \\
\mathrm{I}_{\mathrm{yy}}^{B} \dot{\omega}_{\mathrm{y}}= & \left(\mathrm{I}_{\mathrm{zz}}^{B}-\mathrm{I}_{\mathrm{xx}}^{B}\right) \omega_{\mathrm{x}} \omega_{\mathrm{z}} \\
& +\sum_{i=1}^{4}\left(\left(l_{z i} \alpha_{x i}-l_{x i} \alpha_{z i}\right) u_{1 i}\right) \\
\mathrm{I}_{\mathrm{zz}}^{B} \dot{\omega}_{\mathrm{z}}= & \left(\mathrm{I}_{\mathrm{xx}}^{B}-\mathrm{I}_{\mathrm{yy}}^{B}\right) \omega_{\mathrm{x}} \omega_{\mathrm{y}} \\
& +\sum_{i=1}^{4}\left(\tau_{J i} \mathrm{c}_{\theta_{J i}}+\left(l_{x i} \alpha_{y i}-l_{y i} \alpha_{x i}\right) u_{1 i}\right)
\end{aligned}
$$

3) Quadrotor angular dynamics: Dynamic modeling of quadrotors has been widely discussed in the literature and a detailed analysis can be found in [41]. For notational convenience, let the added propeller speed be

$$
n_{\Sigma i}=\sum_{j=1}^{4}(-1)^{j+1} n_{j}
$$

Taking into account that $\mathrm{I}_{\mathrm{xx}}^{Q}=\mathrm{I}_{\mathrm{yy}}^{Q}$, the differential equations describing the angular motion of the quadrotors in $\mathcal{F}_{Q_{i}}$ become

$$
\begin{aligned}
& \mathrm{I}_{\mathrm{xx}}^{Q} \dot{\omega}_{\mathrm{x} i}=\left(\mathrm{I}_{\mathrm{yy}}^{Q}-\mathrm{I}_{\mathrm{zz}}^{Q}\right) \omega_{\mathrm{y} i} \omega_{\mathrm{z} i}-\mathrm{I}_{\mathrm{zz}}^{P} \omega_{\mathrm{y} i} n_{\Sigma i}+u_{2 i} \\
& \mathrm{I}_{\mathrm{yy}}^{Q} \dot{\omega}_{\mathrm{y} i}=\left(\mathrm{I}_{\mathrm{zz}}^{Q}-\mathrm{I}_{\mathrm{xx}}^{Q}\right) \omega_{\mathrm{x} i} \omega_{\mathrm{z} i}+\mathrm{I}_{\mathrm{zz}}^{P} \omega_{\mathrm{x} i} n_{\Sigma i}+u_{3 i}-\tau_{J i} \mathrm{~s}_{\phi_{J i}} \\
& \mathrm{I}_{\mathrm{zz}}^{Q} \dot{\omega}_{\mathrm{z} i}=u_{4 i}-\tau_{J i} \mathrm{c}_{\phi_{J i}}
\end{aligned}
$$

4) Whole vehicle dynamics: Universal joints constrain the rotational motion of the bodies they connect by imposing the condition of no yaw between them

$$
\psi_{J i}=0 ; \quad \dot{\psi}_{J i}=0 ; \quad \ddot{\psi}_{J i}=0
$$

Due to the assumption that $\phi_{J i}$ and $\theta_{J i}$ are small, this condition can be approximated to

$$
\dot{\omega}_{\mathrm{z} i} \simeq \dot{\omega}_{\mathrm{z}}
$$

For the same reason, in order eliminate constraint torques and obtain a compact model of the system, it can be assumed that

$$
\begin{aligned}
& \tau_{J i} \mathrm{~s}_{\theta_{J i}} \simeq 0 \\
& \tau_{J i} \mathrm{~s}_{\phi_{J i}} \simeq 0 \\
& \tau_{J i}\left(\mathrm{c}_{\theta_{J i}}-\mathrm{c}_{\phi_{J i}}\right) \simeq 0 .
\end{aligned}
$$

At this point it is useful to define forces $\boldsymbol{a}_{1}, \boldsymbol{a}_{2}, \boldsymbol{a}_{3}$ and torques $\boldsymbol{a}_{4}, \boldsymbol{a}_{5}, \boldsymbol{a}_{6}$, named central control actions. They represent the controllable forces and torques that are required to independently act on the six degrees of freedom of the airframe, as depicted in Figure 5.

$$
\begin{aligned}
a_{1} & =\sum_{i=1}^{4} \mathrm{c}_{\phi_{i}} \mathrm{~s}_{\theta_{i}} u_{1 i} \\
a_{2} & =\sum_{i=1}^{4}-\mathbf{s}_{\phi_{i}} u_{1 i} \\
a_{3} & =\sum_{i=1}^{4} \mathrm{c}_{\phi_{i}} \mathrm{c}_{\theta_{i}} u_{1 i} \\
a_{4} & =\sum_{i=1}^{4}\left(l_{y i} \alpha_{z i}-l_{z i} \alpha_{y i}\right) u_{1 i} \\
a_{5} & =\sum_{i=1}^{4}\left(l_{z i} \alpha_{x i}-l_{x i} \alpha_{z i}\right) u_{1 i} \\
a_{6} & =\sum_{i=1}^{4}\left(u_{4 i}+\left(l_{x i} \alpha_{y i}-l_{y i} \alpha_{x i}\right) u_{1 i}\right)
\end{aligned}
$$

Hence, defining system inertia

$$
\mathrm{I}_{\mathrm{zz}}^{S}=\mathrm{I}_{\mathrm{zz}}^{B}+4 \mathrm{I}_{\mathrm{zz}}^{Q}+\sum_{i=1}^{4} m_{i}\left(l_{x i}^{2}+l_{y i}^{2}\right)
$$




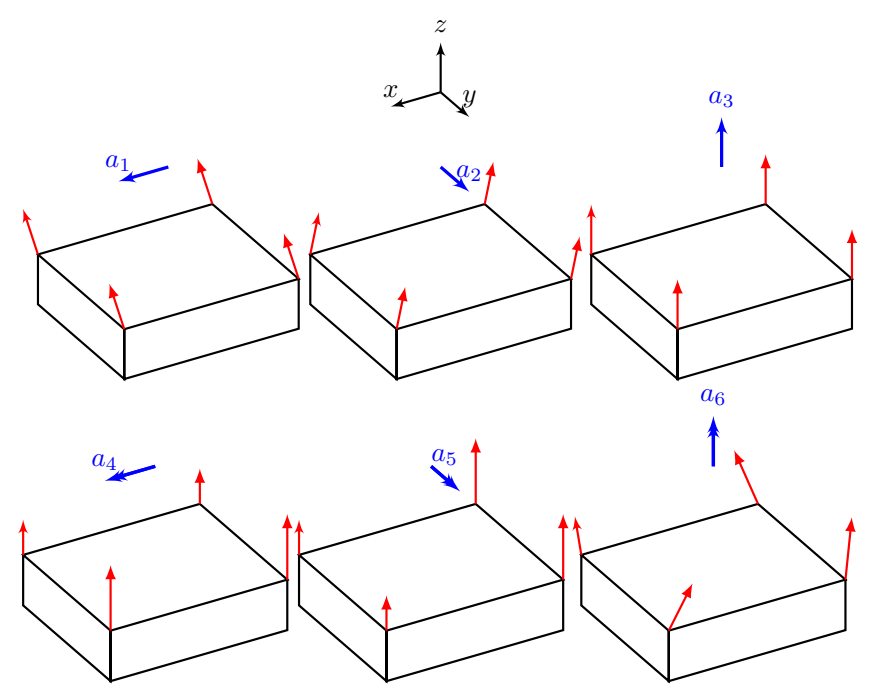

Fig. 5. The six central control actions and the control strategy.

and making use of Equation 3 to determine the Tait-Bryan angles, the equations of motion of the system are:

$$
\left\{\begin{array}{l}
\mathrm{m} \ddot{r}_{\mathrm{X}}=\mathrm{c}_{\psi} a_{1}-\mathrm{s}_{\psi} a_{2} \\
\mathrm{~m} \ddot{r}_{\mathrm{Y}}=\mathrm{s}_{\psi} a_{1}+\mathrm{c}_{\psi} a_{2} \\
\mathrm{~m} \ddot{r}_{\mathrm{Z}}=a_{3}-\mathrm{mg} \\
\mathrm{I}_{\mathrm{xx}}^{B} \dot{\omega}_{\mathrm{x}}=\left(\mathrm{I}_{\mathrm{yy}}^{B}-\mathrm{I}_{\mathrm{zz}}^{B}\right) \omega_{\mathrm{y}} \omega_{\mathrm{z}}+a_{4} \\
\mathrm{I}_{\mathrm{yy}}^{B} \dot{\omega}_{\mathrm{y}}=\left(\mathrm{I}_{\mathrm{zz}}^{B}-\mathrm{I}_{\mathrm{xx}}^{B}\right) \omega_{\mathrm{x}} \omega_{\mathrm{z}}+a_{5} \\
\mathrm{I}_{\mathrm{zz}}^{S} \dot{\omega}_{\mathrm{z}}=\left(\mathrm{I}_{\mathrm{xx}}^{B}-\mathrm{I}_{\mathrm{yy}}^{B}\right) \omega_{\mathrm{x}} \omega_{\mathrm{y}}+a_{6} \\
\mathrm{I}_{\mathrm{xx}}^{Q} \dot{\omega}_{\mathrm{x} i}=\left(\mathrm{I}_{\mathrm{yy}}^{Q}-\mathrm{I}_{\mathrm{zz}}^{Q}\right) \omega_{\mathrm{y} i} \omega_{\mathrm{z} i}-\mathrm{I}_{\mathrm{zz}}^{P} \omega_{\mathrm{y} i} n_{\Sigma i}+u_{2 i} \\
\mathrm{I}_{\mathrm{yy}}^{Q} \dot{\omega}_{\mathrm{y} i}=\left(\mathrm{I}_{\mathrm{zz}}^{Q}-\mathrm{I}_{\mathrm{xx}}^{Q}\right) \omega_{\mathrm{x} i} \omega_{\mathrm{z} i}+\mathrm{I}_{\mathrm{zz}}^{P} \omega_{\mathrm{x} i} n_{\Sigma i}+u_{3 i} \\
\dot{\phi}=\omega_{\mathrm{x}}+\frac{\mathrm{s}_{\phi} \mathrm{s}_{\theta}}{\mathrm{c}_{\theta}} \omega_{\mathrm{y}}+\frac{\mathrm{c}_{\phi} \mathrm{s}_{\theta}}{\mathrm{c}_{\theta}} \omega_{\mathrm{z}} \\
\dot{\theta}=\mathrm{c}_{\phi} \omega_{\mathrm{y}}-\mathrm{s}_{\phi} \omega_{\mathrm{z}} \\
\dot{\psi}=\frac{\mathrm{s}_{\phi} \omega_{\mathrm{y}}+\mathrm{c}_{\phi} \omega_{\mathrm{z}}}{\mathrm{c}_{\theta}} \\
\dot{\phi}_{i}=\omega_{\mathrm{x} i}+\frac{\mathrm{s}_{\phi_{i}} \mathrm{~s}_{\theta_{i}}}{\mathrm{c}_{\theta_{i}}} \omega_{\mathrm{y}_{i}}+\frac{\mathrm{c}_{\phi_{i}} \mathrm{~s}_{\theta_{i}}}{\mathrm{c}_{\theta_{i}}} \omega_{\mathrm{z} i} \\
\dot{\theta}_{i}=\mathrm{c}_{\phi_{i}} \omega_{\mathrm{y}_{i}}-\mathrm{s}_{\phi_{i}} \omega_{\mathrm{z} i}
\end{array}\right.
$$

for $i=1, \ldots, 4$.

Equations 8, 23 and 25 constitute a dynamic model of the system that take as input sixteen motor speeds $n_{i j}(t)$ and, for given initial conditions, fully specify the motion of the system with the adopted simplifications.

\section{CONTROL}

Throughout the remaining sections subscript $\{\cdot\}_{r}$ and $\{\cdot\}_{m}$ will denote a reference and a measurement signal respectively.

Although, due to its overactuated and nonlinear nature, the system is suitable for the development of nonlinear and optimization-based controllers, in this paper a linear control architecture will be presented, demonstrating that a PID based algorithm is enough to control the system along simple trajectories.

\section{A. Control architecture}

The here described aerial vehicle has a distributed nature: actuators and sensors required for its stabilization are spread along the plant. A question that arises in this kind of systems is whether to centralize or decentralize the information processing (i.e. the control). In this paper, a centralized hierarchical control has been adopted: a central controller coordinates four peripheral controllers, which send references to a total of sixteen Electronic Speed Controllers (ESC), as depicted in Figure 6. The communication between controllers is unidirectional and it is done by means of Pulse Width Modulation (PWM) signals.

Both the central and the peripheral controllers run a modified version of the PX4 middleware [43] and they are equipped with an Inertial Measurement Unit (IMU) that measures translational acceleration and rotational speeds, as well as a magnetometer and a barometer. Besides, the central controller is connected to a LIDAR Lite v3 distance sensor and an optical flow camera [44]. Making use of an Extended Kalman Filter, the sensor information is fused to estimate the position and orientation of the airframe and the four peripheral quadrotors.

\section{B. Central control}

The central controller receives the desired trajectory as a set of airframe poses $\left(r_{X, r}, r_{Y, r}, r_{Z, r}, \phi_{r}, \theta_{r}, \psi_{r}\right)$ and it must compute required forces and torques $\left(a_{1, \ldots, 6}\right)$ to ensure good tracking. In order to do so, one nested PID loop is implemented for each degree of freedom.

$$
\begin{aligned}
\dot{r}_{x, r}= & k_{p, r_{x}}\left(r_{x, r}-r_{x, m}\right) \\
\dot{r}_{y, r}= & k_{p, r_{y}}\left(r_{y, r}-r_{y, m}\right) \\
\omega_{\mathrm{x} r}= & k_{p, \phi}\left(\phi_{r}-\phi_{m}\right) \\
\omega_{\mathrm{y}_{r}}= & k_{p, \theta}\left(\theta_{r}-\theta_{m}\right) \\
\omega_{\mathrm{z} r}= & k_{p, \psi}\left(\psi_{r}-\psi_{m}\right) \\
a_{1}= & k_{p, v_{x}}\left(\dot{r}_{x, r}-\dot{r}_{x, m}\right)+k_{i, v_{x}} \int\left(\dot{r}_{x, r}-\dot{r}_{x, m}\right) \mathrm{d} t \\
& k_{d, v_{x}} \mathrm{~d}\left(\dot{r}_{x, r}-\dot{r}_{x, m}\right) / \mathrm{d} t \\
a_{2}= & k_{p, v_{y}}\left(\dot{r}_{y, r}-\dot{r}_{y, m}\right)+k_{i, v_{y}} \int\left(\dot{r}_{y, r}-\dot{r}_{y, m}\right) \mathrm{d} t \\
& k_{d, v_{y}} \mathrm{~d}\left(\dot{r}_{y, r}-\dot{r}_{y, m}\right) / \mathrm{d} t \\
a_{3}= & k_{p, z}\left(r_{z, r}-r_{z, m}\right)+k_{i, z} \int\left(r_{z, r}-r_{z, m}\right) \mathrm{d} t \\
a_{4}= & k_{p, \omega_{\mathrm{x}}}\left(\omega_{\mathrm{x} r}-\omega_{\mathrm{x} m}\right)+k_{i, \omega_{\mathrm{x}}} \int\left(\omega_{\mathrm{x} r}-\omega_{\mathrm{x} m}\right) \mathrm{d} t \\
a_{5}= & k_{p, \omega_{\mathrm{y}}}\left(\omega_{\mathrm{y}_{r}}-\omega_{\mathrm{y} m}\right)+k_{i, \omega_{\mathrm{y}}} \int\left(\omega_{\mathrm{y}_{r}}-\omega_{\mathrm{y} m}\right) \mathrm{d} t \\
a_{6}= & k_{p, \omega_{\mathrm{z}}}\left(\omega_{\mathrm{z} r}-\omega_{\mathrm{z} m}\right)+k_{i, \omega_{\mathrm{z}}} \int\left(\omega_{\mathrm{z} r}-\omega_{\mathrm{z} m}\right) \mathrm{d} t
\end{aligned}
$$

where

$$
\begin{array}{lll}
r_{x}=r_{\mathrm{X}} \mathrm{c}_{\psi}+r_{\mathrm{Y}} \mathrm{s}_{\psi} & r_{y}=-r_{\mathrm{X}} \mathbf{s}_{\psi}+r_{\mathrm{Y}} \mathrm{c}_{\psi} & r_{z}=r_{Z} \\
\dot{r}_{x}=\dot{r}_{\mathrm{X}} \mathrm{c}_{\psi}+\dot{r}_{\mathrm{Y}} \mathrm{s}_{\psi} & \dot{r}_{y}=-\dot{r}_{\mathrm{X}} \mathbf{s}_{\psi}+\dot{r}_{\mathrm{Y}} \mathrm{c}_{\psi} & \dot{r}_{z}=\dot{r}_{Z}
\end{array}
$$

It is important to remark that it is also possible to semimanually control the aerial vehicle by means of an RC transmitter. The only modification to the control algorithm is the substitution of the commanded pose with the following inputs: $a_{1}, a_{2}, a_{3}, \phi_{r}, \theta_{r}, \omega_{\mathrm{zr} r}$. Thus, translational motion is directly controlled by the human pilot and the central controller is responsible of maintaining the desired values of pitch, roll and yaw rate.

Once the desired forces and moments are computed, they must be transformed into appropriate commands for the 


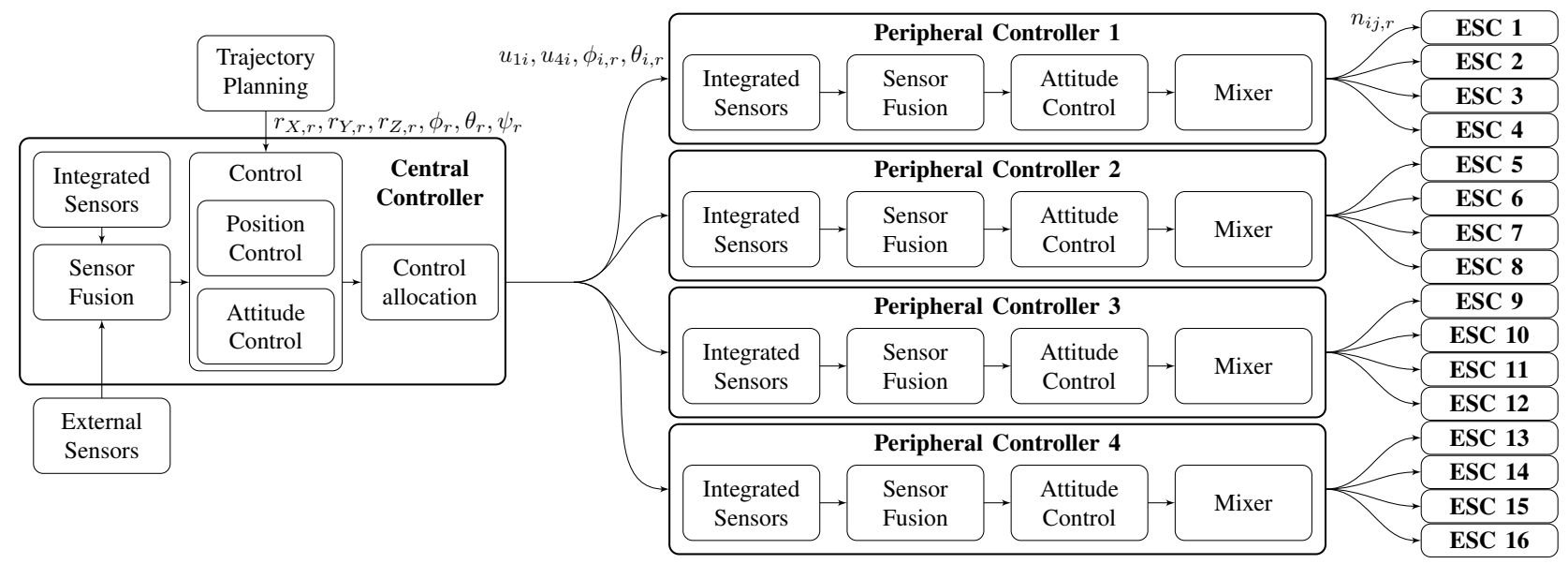

Fig. 6. Diagram of the control architecture. Sensing, information processing and actuation is distributed along the system. The control happens in a hierarchical way: in order to follow a given trajectory $\left(r_{X, r}, r_{Y, r}, r_{Z, r}, \phi_{r}, \theta_{r}, \psi_{r}\right)$ the central controller computes the reference of the peripheral controllers $\left(u_{1 i}, u_{4 i}, \phi_{i, r}, \theta_{i, r}\right)$ and these compute the reference of the Electronic Speed Controllers (ESC) $\left(n_{i j, r}\right)$ which directly act on the system.

quadrotors. However, Equation 23 shows that there are many different ways of affecting each control action and that they are nonlinearly coupled. Thus, a control allocation rule is required to translate $a_{1, \ldots, 6}$ into $u_{1 i}, u_{4 i}, \phi_{i, r}$ and $\theta_{i, r}$.

\section{Central control allocation}

For the sake of simplicity, the space of possible control laws will be limited by establishing that quadrotors will not make use of their torque generating capacity,

$$
u_{4 i}=0 .
$$

In practice, the torques transmitted by quadrotors to the airframe will not be zero due to nonidealities and unmodeled dynamics but they can be treated as disturbances.

Hence, the control allocation strategy will consist in conveniently modifying $u_{1 i}, i=1, \ldots, 4$ to achieve the desired $a_{3}, a_{4}$ and $a_{5}$ and varying $\phi_{i}$ and $\theta_{i}, i=1, \ldots, 4$ to affect $a_{1}, a_{2}$ and $a_{6}$. The control effort is equally distributed among the quadrotors as depicted in Figure 5

$$
\begin{aligned}
& {\left[\begin{array}{l}
u_{11} \\
u_{12} \\
u_{13} \\
u_{14}
\end{array}\right]=\left[\begin{array}{ccc}
\beta & \gamma & -\gamma \\
\beta & \gamma & \gamma \\
\beta & -\gamma & \gamma \\
\beta & -\gamma & -\gamma
\end{array}\right]\left[\begin{array}{l}
a_{3} \\
a_{4} \\
a_{5}
\end{array}\right]} \\
& {\left[\begin{array}{c}
\phi_{1, r} \\
\phi_{2, r} \\
\phi_{3, r} \\
\phi_{4, r} \\
\theta_{1, r} \\
\theta_{2, r} \\
\theta_{3, r} \\
\theta_{4, r}
\end{array}\right]=\left[\begin{array}{ccc}
0 & -\delta & -\epsilon \\
0 & -\delta & \epsilon \\
0 & -\delta & \epsilon \\
0 & -\delta & -\epsilon \\
\delta & 0 & -\epsilon \\
\delta & 0 & -\epsilon \\
\delta & 0 & \epsilon \\
\delta & 0 & \epsilon
\end{array}\right]\left[\begin{array}{l}
a_{1} \\
a_{2} \\
a_{6}
\end{array}\right]}
\end{aligned}
$$

where $\beta, \gamma, \delta$ and $\epsilon$ are proportionality gains.

\section{Peripheral control}

The task of the peripheral controller is to follow the references $\left(\phi_{r}\right.$ and $\left.\theta_{r}\right)$ commanded by the central one. In order to do so, it makes use of the following cascade controllers:

$$
\begin{aligned}
\omega_{\mathrm{x} i, r}= & k_{p, \phi_{i}}\left(\phi_{i, r}-\phi_{i, m}\right) \\
\omega_{\mathrm{y}_{i, r}}= & k_{p, \theta_{i}}\left(\theta_{i, r}-\theta_{i, m}\right) \\
u_{2 i}= & k_{p, \omega_{\mathrm{x}}}\left(\omega_{\mathrm{x} i, r}-\omega_{\mathrm{x} i, m}\right)+k_{i, \omega_{\mathrm{x} i}} \int\left(\omega_{\mathrm{x} i, r}-\omega_{\mathrm{x} i, m}\right) \mathrm{d} t \\
& +k_{d, \omega_{\mathrm{x} i}} \mathrm{~d}\left(\omega_{\mathrm{x} i, r}-\omega_{\mathrm{x} i, m}\right) / \mathrm{d} t \\
u_{3 i}= & k_{p, \omega_{\mathrm{y}}}\left(\omega_{\mathrm{y}_{i, r}}-\omega_{\mathrm{y}_{i, m}}\right)+k_{i, \omega_{\mathrm{y}}} \int\left(\omega_{\mathrm{y}_{i, r}}-\omega_{\mathrm{y}_{i, m}}\right) \mathrm{d} t \\
& +k_{d, \omega_{\mathrm{y}}} \mathrm{d}\left(\omega_{\mathrm{y}_{i, r}}-\omega_{\mathrm{y}_{i, m}}\right) / \mathrm{d} t
\end{aligned}
$$

Peripheral control allocation is done by what is known as the Mixer: the algorithm that, taking the desired $u_{h i}$ computes, with Equation 8, the required motor angular speed references $n_{i j, r}$, which are commanded to the ESCs.

\section{EXPERIMENTAL RESUlTS}

The following section presents some preliminary experimental results that show the capabilities of the architecture.

\section{A. Experimental setup}

A prototype of dimensions $1.6 \times 2.16 \times 1.2 \mathrm{~m}$ was built to test the proposed vehicle architecture (see Figure 7). It consists of five Pixhawk 4 controllers, sixteen T-motor ALPHA-40A LV ESCs, sixteen T-motor U3 $700 \mathrm{KV}$ motors and sixteen $T$-motor $13 \times 4.4$ propellers. The power train makes use of four Turnigy 5.0 $6 \mathrm{~S}$ batteries and the control is powered independently by means of a Turnigy $5.02 \mathrm{~S}$ battery. The mechanical structure is based on carbon fiber tubes, aluminum parts and 3D printed plastic parts, and the total vehicle weight is $28.6 \mathrm{~kg}$.

The control algorithm runs at a sample time of $5 \mathrm{~ms}$ and all controller gains are chosen on a trial and error basis. As a general rule, first, inner control loops are stabilized by means of proportional gains, then outer loops. Once the system is stable, integral terms are introduced to compensate for stationary-state errors and then derivatives are introduced 


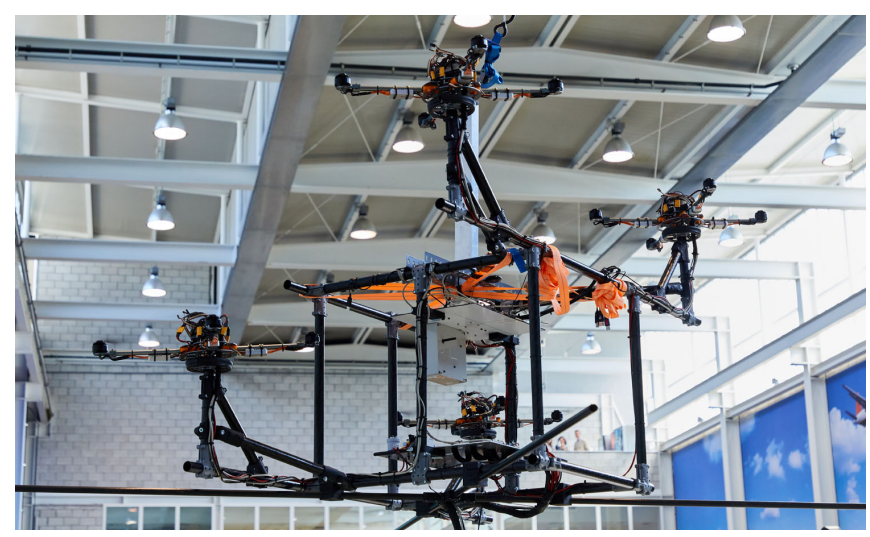

Fig. 7. Picture of the prototype in tethered flight inside Tecnalia's facilities.

TABLE I

SELECTED GAINS FOR THE NESTED PID CONTROLLERS.

\begin{tabular}{ccccccc}
\hline & \multicolumn{3}{c}{ Position } & \multicolumn{3}{c}{ Speed } \\
\hline & $k_{p}$ & $k_{i}$ & $k_{d}$ & $k_{p}$ & $k_{i}$ & $k_{d}$ \\
\hline$r_{x}$ & 1 & 0 & 0 & 0.38 & 0.03 & 0.03 \\
$r_{y}$ & 1 & 0 & 0 & 0.38 & 0.03 & 0.03 \\
$r_{z}$ & 0.2 & 0.02 & 0 & $\varnothing$ & $\varnothing$ & $\varnothing$ \\
$\phi$ & 4 & 0 & 0 & 0.23 & 0.10 & 0 \\
$\theta$ & 4 & 0 & 0 & 0.23 & 0.10 & 0 \\
$\psi$ & 3 & 0 & 0 & 0.22 & 0.06 & 0 \\
$\phi_{i}$ & 3.5 & 0 & 0 & 0.06 & 0.05 & 0.003 \\
$\theta_{i}$ & 3.5 & 0 & 0 & 0.06 & 0.05 & 0.003 \\
\hline
\end{tabular}

to smooth system response. The tuning process began in simulation and was then adjusted with the physical system, eventually leading to the set of controller gains listed in Table I (symbol $\varnothing$ indicates that there is no inner loop in $\left.r_{z}\right)$. For simplicity, the control allocation parameters $\beta, \gamma, \delta$ and $\epsilon$ were all set to 1 .

\section{B. Autonomous trajectory tracking}

Due to the lack of an external motion capture system to accurately track the vehicle's full pose, only on-board sensors (IMU, LIDAR and optical flow) were employed to validate the performance of the control algorithm. This approach limited the complexity of the trajectories that could be safely tested. Thus, the chosen autonomous trajectory was a sinusoid in the $y_{E}$ axis while keeping all other variables constant. Even if it is a very simple maneuver, it is not possible to follow it with parallel axis multirotors because of their underactuation. Figure 8 shows the results of the experiment, proving that it is capable of independently controlling the six degrees of freedom of the airframe. Table II quantifies the tracking performance by means of the Root Mean Square Error (RMSE).

$$
\operatorname{RMSE}(\cdot)=\sqrt{\frac{\sum_{k=1}^{N}\left(\{\cdot\}_{m, k}-\{\cdot\}_{r, k}\right)^{2}}{N}}
$$

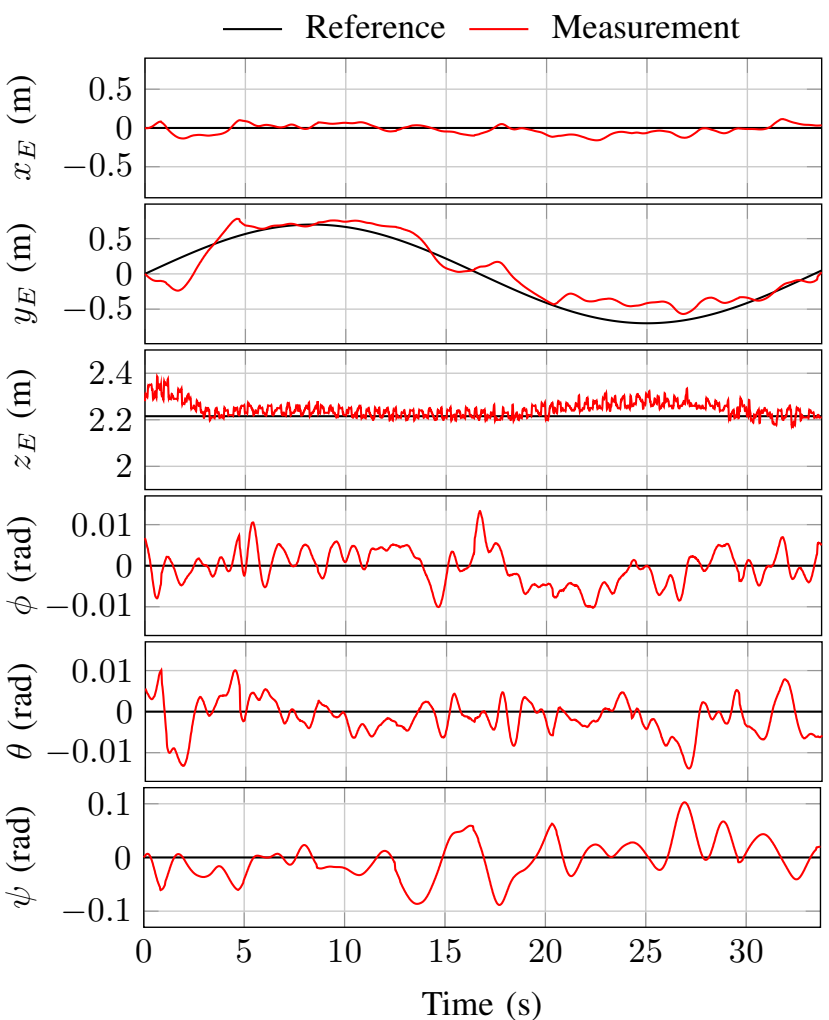

Fig. 8. Experimental results: the six degrees of freedom of the airframe are controlled independently, following a sinusoid in $y_{E}$ while keeping the rest of the signals constant. The measurements are done with on-board sensors (IMU, LIDAR and optical flow).

TABLE II

Tracking PERFormance: RoOT MeAN SQuare ERror

\begin{tabular}{cccccc}
\hline$x_{E}(\mathrm{~m})$ & $y_{E}(\mathrm{~m})$ & $z_{E}(\mathrm{~m})$ & $\phi(\mathrm{rad})$ & $\theta(\mathrm{rad})$ & $\psi(\mathrm{rad})$ \\
\hline 0.067 & 0.172 & 0.044 & 0.004 & 0.005 & 0.037 \\
\hline
\end{tabular}

\section{CONCLUSions}

We have presented an overactuated aerial platform that does not rely on inefficient propeller configurations or dedicated rotor-tilting actuators. A simplified dynamic model of the system has been derived using Newton-Euler formalism and a linear control strategy, capable of decoupling translational and rotational motion has been proposed. Preliminary experimental results with a prototype have shown the feasibility of the concept and its ability to independently control the six degrees of freedom of the airframe.

We believe that the proposed architecture is particularly well suited for tasks where physical interaction with the environment is required: it is maneuverable, redundant and, due to its modularity, it can be scaled up, further increasing its payload. Thus, it has the potential to improve activities such as work in hazardous conditions, manufacture or transport of goods and people.

Future work will focus on developing a more robust control algorithm, optimizing the control allocation strategy and improving the experimental setup to fully exploit the capabilities of the architecture within demanding applications. 


\section{ACKNOWLEDGMENT}

The authors would like to thank the following people for their contributions and support (in alphabetic order): Leire Abad, Iñigo Eguizabal, Jose Ramón García, Eduardo Guzmán, Mikel Lizeaga, Arkaitz Oyarzabal, Alexandre Paris, Andrés Sierra, Tim Smithers and Haritz Vallejo.

\section{REFERENCES}

[1] H. Bonyan Khamseh, F. Janabi-Sharifi, and A. Abdessameud, "Aerial manipulation-a literature survey," Robotics and Autonomous Systems, vol. 107, pp. 221-235, Sep 2018. [Online]. Available: https://doi.org/10.1016/j.robot.2018.06.012

[2] F. Ruggiero, V. Lippiello, and A. Ollero, "Aerial manipulation: A literature review," IEEE Robotics and Automation Letters, vol. 3, no. 3, pp. 1957-1964, Jul 2018. [Online]. Available: http://doi.org/10.1109/LRA.2018.2808541

[3] A. Jimenez-Cano, J. Martin, G. Heredia, A. Ollero, and R. Cano, "Control of an aerial robot with multi-link arm for assembly tasks," in 2013 IEEE International Conference on Robotics and Automation. IEEE, 2013, pp. 4916-4921. [Online]. Available: http://doi.org/10.1109/icra.2013.6631279

[4] T. W. Danko and P. Y. Oh, "Design and control of a hyper-redundant manipulator for mobile manipulating unmanned aerial vehicles," Journal of Intelligent \& Robotic Systems, vol. 73, no. 1-4, pp. 709-723, 2014. [Online]. Available: https://doi.org/10.1007/s10846-013-9935-2

[5] T. W. Danko, K. P. Chaney, and P. Y. Oh, "A parallel manipulator for mobile manipulating uavs," in 2015 IEEE international conference on technologies for practical robot applications (TePRA). IEEE, 2015, pp. 1-6. [Online]. Available: http://doi.org/10.1109/tepra.2015. 7219682

[6] F. Ruggiero, M. A. Trujillo, R. Cano, H. Ascorbe, A. Viguria, C. Peréz, V. Lippiello, A. Ollero, and B. Siciliano, "A multilayer control for multirotor uavs equipped with a servo robot arm," in 2015 IEEE international conference on robotics and automation (ICRA). IEEE, 2015, pp. 4014-4020. [Online]. Available: http: //doi.org/10.1109/icra.2015.7139760

[7] A. Suarez, A. Jimenez-Cano, V. Vega, G. Heredia, A. RodriguezCastaño, and A. Ollero, "Lightweight and human-size dual arm aerial manipulator," in 2017 International Conference on Unmanned Aircraft Systems (ICUAS). IEEE, 2017, pp. 1778-1784. [Online]. Available: http://doi.org/10.1109/icuas.2017.7991357

[8] B. Crowther, A. Lanzon, M. Maya-Gonzalez, and D. Langkamp, "Kinematic analysis and control design for a nonplanar multirotor vehicle," Journal of Guidance, Control, and Dynamics, vol. 34, no. 4, pp. 1157-1171, 2011. [Online]. Available: http://doi.org/10.2514/1. 51186

[9] G. Jiang and R. Voyles, "Hexrotor uav platform enabling dextrous interaction with structures-flight test," in 2013 IEEE international symposium on safety, security, and rescue robotics (SSRR). IEEE, 2013, pp. 1-6. [Online]. Available: http://doi.org/10.1109/ssrr.2013. 6719377

[10] S. Rajappa, M. Ryll, H. H. Bülthoff, and A. Franchi, "Modeling, control and design optimization for a fully-actuated hexarotor aerial vehicle with tilted propellers," in 2015 IEEE international conference on robotics and automation (ICRA). IEEE, 2015, pp. 4006-4013. [Online]. Available: http://doi.org/10.1109/icra.2015.7139759

[11] P. Roque and R. Ventura, "Space cobot: Modular design of an holonomic aerial robot for indoor microgravity environments," in 2016 IEEE/RSJ International Conference on Intelligent Robots and Systems (IROS). IEEE, 2016, pp. 4383-4390. [Online]. Available: http://doi.org/10.1109/iros.2016.7759645

[12] M. Ryll, G. Muscio, F. Pierri, E. Cataldi, G. Antonelli, F. Caccavale, and A. Franchi, " $6 \mathrm{~d}$ physical interaction with a fully actuated aerial robot," in 2017 IEEE International Conference on Robotics and Automation (ICRA). IEEE, 2017, pp. 5190-5195. [Online]. Available: http://doi.org/10.1109/icra.2017.7989608

[13] E. Kaufman, K. Caldwell, D. Lee, and T. Lee, "Design and development of a free-floating hexrotor uav for 6-dof maneuvers," in 2014 IEEE Aerospace Conference. IEEE, 2014, pp. 1-10. [Online]. Available: http://doi.org/10.1109/aero.2014.6836427
[14] N. Staub, D. Bicego, Q. Sablé, V. Arellano, S. Mishra, and A. Franchi, "Towards a flying assistant paradigm: The othex," in 2018 IEEE International Conference on Robotics and Automation (ICRA). IEEE, 2018, pp. 6997-7002. [Online]. Available: http: //doi.org/10.1109/icra.2018.8460877

[15] H. Romero, S. Salazar, and R. Lozano, "Real-time stabilization of an eight-rotor uav using optical flow," IEEE Transactions on Robotics, vol. 25, no. 4, pp. 809-817, 2009. [Online]. Available: http://doi.org/10.1109/TRO.2009.2018972

[16] F. von Frankenberg and S. B. Nokleby, "Inclined landing testing of an omni-directional unmanned aerial vehicle," Transactions of the Canadian Society for Mechanical Engineering, vol. 42, no. 1, pp. 6170, 2018. [Online]. Available: http://doi.org/10.1139/tcsme-2017-0008

[17] A. Nikou, G. C. Gavridis, and K. J. Kyriakopoulos, "Mechanical design, modelling and control of a novel aerial manipulator," in 2015 IEEE International Conference on Robotics and Automation (ICRA). IEEE, 2015, pp. 4698-4703. [Online]. Available: http: //doi.org/10.1109/icra.2015.7139851

[18] D. Brescianini and R. D'Andrea, "An omni-directional multirotor vehicle," Mechatronics, vol. 55, pp. 76-93, 2018. [Online]. Available: http://doi.org/10.1016/j.mechatronics.2018.08.005

[19] S. Park, J. Lee, J. Ahn, M. Kim, J. Her, G.-H. Yang, and D. Lee, "Odar: Aerial manipulation platform enabling omnidirectional wrench generation," IEEE/ASME Transactions on mechatronics, vol. 23, no. 4, pp. 1907-1918, 2018. [Online]. Available: http: //doi.org/10.1109/tmech.2018.2848255

[20] M. Tognon and A. Franchi, "Omnidirectional aerial vehicles with unidirectional thrusters: Theory, optimal design, and control," IEEE Robotics and Automation Letters, vol. 3, no. 3, pp. 2277-2282, 2018.

[21] D. Mellinger, M. Shomin, N. Michael, and V. Kumar, Cooperative Grasping and Transport Using Multiple Quadrotors, ser. Springer Tracts in Advanced Robotics. Springer Berlin Heidelberg, 2013, vol. 83, ch. chapter 39, pp. 545-558. [Online]. Available: http://doi.org/10.1007/978-3-642-32723-0_39

[22] Y. H. Tan, S. Lai, K. Wang, and B. M. Chen, "Cooperative control of multiple unmanned aerial systems for heavy duty carrying," Annual Reviews in Control, vol. 46, pp. 44 - 57, 2018. [Online]. Available: http://doi.org/10.1016/j.arcontrol.2018.07.001

[23] C. Papachristos, K. Alexis, and A. Tzes, "Efficient force exertion for aerial robotic manipulation: Exploiting the thrust-vectoring authority of a tri-tiltrotor uav," in 2014 IEEE international conference on robotics and automation (ICRA). IEEE, 2014, pp. 4500-4505. [Online]. Available: http://doi.org/10.1109/icra.2014.6907516

[24] Y. Long and D. J. Cappelleri, "Omnicopter: A novel overactuated micro aerial vehicle," in Advances in Mechanisms, Robotics and Design Education and Research. Springer, 2013, pp. 215-226. [Online]. Available: http://doi.org/10.1007/978-3-319-00398-6_16

[25] M. Ryll, D. Bicego, and A. Franchi, "Modeling and control of fast-hex: A fully-actuated by synchronized-tilting hexarotor," in 2016 IEEE/RSJ International Conference on Intelligent Robots and Systems (IROS). IEEE, 2016, pp. 1689-1694. [Online]. Available: http://doi.org/10.1109/iros.2016.7759271

[26] M. Ryll, H. H. Bülthoff, and P. R. Giordano, "A novel overactuated quadrotor unmanned aerial vehicle: Modeling, control, and experimental validation," IEEE Transactions on Control Systems Technology, vol. 23, no. 2, pp. 540-556, 2015. [Online]. Available: http://doi.org/10.1109/tcst.2014.2330999

[27] A. Oosedo, S. Abiko, S. Narasaki, A. Kuno, A. Konno, and M. Uchiyama, "Flight control systems of a quad tilt rotor unmanned aerial vehicle for a large attitude change," in 2015 IEEE International Conference on Robotics and Automation (ICRA). IEEE, 2015, pp. 2326-2331. [Online]. Available: http://doi.org/10.1109/icra.2015. 7139508

[28] M. Kamel, S. Verling, O. Elkhatib, C. Sprecher, P. Wulkop, Z. Taylor, R. Siegwart, and I. Gilitschenski, "The voliro omniorientational hexacopter: An agile and maneuverable tiltable-rotor aerial vehicle," IEEE Robotics \& Automation Magazine, vol. 25, no. 4, pp. 34-44, Dec 2018. [Online]. Available: http://doi.org/10.1109/mra.2018.2866758

[29] P. Segui-Gasco, Y. Al-Rihani, H.-S. Shin, and A. Savvaris, "A novel actuation concept for a multi rotor uav," Journal of Intelligent \& Robotic Systems, vol. 74, no. 1-2, pp. 173-191, 2014. [Online]. Available: https://doi.org/10.1007/s10846-013-9987-3

[30] M. Odelga, P. Stegagno, and H. H. Bülthoff, "A fully actuated quadrotor uav with a propeller tilting mechanism: Modeling and control," in 2016 IEEE international conference on advanced 
intelligent mechatronics (AIM). IEEE, 2016, pp. 306-311. [Online]. Available: http://doi.org/10.1109/aim.2016.7576784

[31] M. Nigro, F. Pierri, and F. Caccavale, "Preliminary design, modeling and control of a fully actuated quadrotor uav," in 2019 International Conference on Unmanned Aircraft Systems (ICUAS). IEEE, 2019, pp. 1108-1116. [Online]. Available: http: //doi.org/10.1109/icuas.2019.8798092

[32] N. Michael, J. Fink, and V. Kumar, "Cooperative manipulation and transportation with aerial robots," Autonomous Robots, vol. 30, no. 1, pp. 73-86, 2011. [Online]. Available: http://doi.org/10.1007/ s10514-010-9205-0

[33] R. Ritz, M. W. Müller, M. Hehn, and R. D'Andrea, "Cooperative quadrocopter ball throwing and catching," in 2012 IEEE/RSJ International Conference on Intelligent Robots and Systems. IEEE, 2012, pp. 4972-4978. [Online]. Available: http://doi.org/10.1109/iros. 2012.6385963

[34] K. Sreenath and V. Kumar, "Dynamics, control and planning for cooperative manipulation of payloads suspended by cables from multiple quadrotor robots," Proceedings of the Robotics: Science and Systems Conference (RSS), vol. 1, no. r2, p. r3, 2013. [Online]. Available: http://doi.org/10.15607/rss.2013.ix.011

[35] M. Manubens Ferriol, D. Devaurs, L. Ros Giralt, and J. Cortés, "Motion planning for 6d manipulation with aerial towed-cable systems," in Proceedings of the Robotics: Science and Systems Conference (RSS), 2013. [Online]. Available: http://doi.org/10.15607/ rss.2013.ix.028

[36] M. Gassner, T. Cieslewski, and D. Scaramuzza, "Dynamic collaboration without communication: Vision-based cable-suspended load transport with two quadrotors," in 2017 IEEE International Conference on Robotics and Automation (ICRA). IEEE, 2017, pp. 5196-5202. [Online]. Available: http://doi.org/10.1109/icra.2017. 7989609

[37] T. Lee, K. Sreenath, and V. Kumar, "Geometric control of cooperating multiple quadrotor uavs with a suspended payload," in 52nd IEEE Conference on Decision and Control, Dec 2013, pp. 5510-5515. [Online]. Available: http://doi.org/10.1109/cdc.2013.6760757
[38] D. Six, S. Briot, A. Chriette, and P. Martinet, "The kinematics, dynamics and control of a flying parallel robot with three quadrotors," IEEE Robotics and Automation Letters, vol. 3, no. 1, pp. 559-566, Jan 2018. [Online]. Available: http://doi.org/10.1109//ra.2017.2774920

[39] H.-N. Nguyen, S. Park, J. Park, and D. Lee, "A novel robotic platform for aerial manipulation using quadrotors as rotating thrust generators," IEEE Transactions on Robotics, vol. 34, no. 2, pp. 353-369, Apr 2018. [Online]. Available: http://doi.org/10.1109/tro.2018.2791604

[40] A. Tagliabue, M. Kamel, R. Siegwart, and J. Nieto, "Robust collaborative object transportation using multiple mavs," The International Journal of Robotics Research, vol. 38, no. 9, pp. 1020-1044, Aug 2019. [Online]. Available: http://doi.org/10.1177/ 0278364919854131

[41] S. Bouabdallah, "Design and control of quadrotors with application to autonomous flying," Ph.D. dissertation, École Polytechnique Fédérale de Lausanne (EPFL), Lausanne, 2007. [Online]. Available: http://doi.org/10.5075/epfl-thesis-3727

[42] B. Etkin and L. D. Reid, Dynamics of flight. Wiley New York, 1959 , vol. 2.

[43] L. Meier, D. Honegger, and M. Pollefeys, "Px4: A node-based multithreaded open source robotics framework for deeply embedded platforms," in 2015 IEEE International Conference on Robotics and Automation (ICRA), May 2015, pp. 6235-6240. [Online]. Available: http://doi.org/10.1109/icra.2015.7140074

[44] D. Honegger, L. Meier, P. Tanskanen, and M. Pollefeys, "An open source and open hardware embedded metric optical flow cmos camera for indoor and outdoor applications," in 2013 IEEE International Conference on Robotics and Automation (ICRA), May 2013, pp. 17361741. [Online]. Available: http://doi.org/10.1109/icra.2013.6630805 